\title{
A case of metastatic testicular germ-cell tumor with rhabdomyosarcoma successfully treated with germ-cell tumor-oriented chemotherapy
}

\author{
Makoto Kagawa Toshiki Kijima Yasuhisa Fujii - Minato Yokoyama • \\ Junichiro Ishioka $\cdot$ Yoh Matsuoka Noboru Numao Kazutaka Saito • \\ Fumitaka Koga $\cdot$ Hitoshi Masuda $\cdot$ Kazunori Kihara
}

Received: 8 September 2011 / Accepted: 1 December 2011/Published online: 20 December 2011

(C) The Japan Society of Clinical Oncology 2011

\begin{abstract}
We report a case of metastatic testicular germcell tumor (GCT) with malignant transformation (MT) successfully treated with a GCT-oriented chemotherapy followed by surgery. A 48-year-old man presented with a right testicular tumor with retroperitoneal lymph node metastases. Pathological examination revealed that the primary tumor was rhabdomyosarcoma with mature teratoma, and the rhabdomyosarcoma element expressed placental alkaline phosphatase (PLAP) which is a marker of primitive germ cells or GCTs. After 4 cycles of GCT-oriented chemotherapy (VIP: etoposide, ifosfamide, and cisplatin), radiological partial response of retroperitoneal metastases was achieved, and subsequent retroperitoneal lymph node dissection revealed no remaining tumor cells. Our report suggests that GCT-oriented chemotherapy may be effective in treatment of patients with metastatic GCT with MT, particularly tumors which express PLAP in the MT element, although this disease entity has traditionally been considered to be chemoresistant.
\end{abstract}

Keywords Germ-cell tumor - Malignant transformation · Chemotherapy

\section{Introduction}

Germ-cell tumors (GCTs) are composed of a variety of histological subtypes usually classified as pure seminoma or

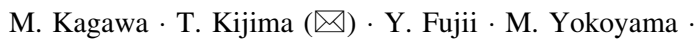

J. Ishioka - Y. Matsuoka - N. Numao - K. Saito - F. Koga ·

H. Masuda $\cdot$ K. Kihara

Department of Urology, Tokyo Medical and Dental

University Graduate School, 1-5-45 Yushima,

Bunkyo-ku, Tokyo 113-8510, Japan

e-mail: toshiki.uro@tmd.ac.jp nonseminomatous GCTs. On rare occasions, GCT cells undergo somatic malignant transformation (MT). This phenomenon, also known as 'teratoma with MT' according to the World Health Organization (WHO) definition [1], occurs in up to $6 \%$ of patients with GCTs [2-4]. The prognosis of patients with MT has been reported to be poorer than that of patients with the GCT counterpart [2-8]. The chemoresistance of the MT element has been considered to be one of the main reasons for the negative prognostic impact of MT. To overcome this problem, several authors have advocated the use of MT-adapted chemotherapy, but its reported efficacy has been unconvincing [5, $7,8]$. Here, we report a case of metastatic testicular teratoma with rhabdomyosarcoma in which pathological complete remission of retroperitoneal lymph node metastases was achieved through GCT-oriented chemotherapy and a disease-free state was maintained for more than 16 months following subsequent surgical resection.

\section{Case report}

A 48-year-old man was referred to our hospital with a right scrotal mass. Ultrasound examination showed a heteroechoic tumor in the right testis (Fig. 1a). Computed tomography (CT) scan revealed retroperitoneal lymph node metastases up to $2.0 \mathrm{~cm}$ in size (Fig. 1b). No visceral metastases were observed. The patient's serum levels of $\alpha$ fetoprotein (AFP), human chorionic gonadotropin (hCG), $\beta$-hCG, and lactic acid dehydrogenase were within normal range.

The patient underwent right orchiectomy. The tumor showed a solid white to yellow cut surface with focal areas of hemorrhage (Fig. 2a). Histological examination revealed that more than $90 \%$ of the tumor lesion was composed of 

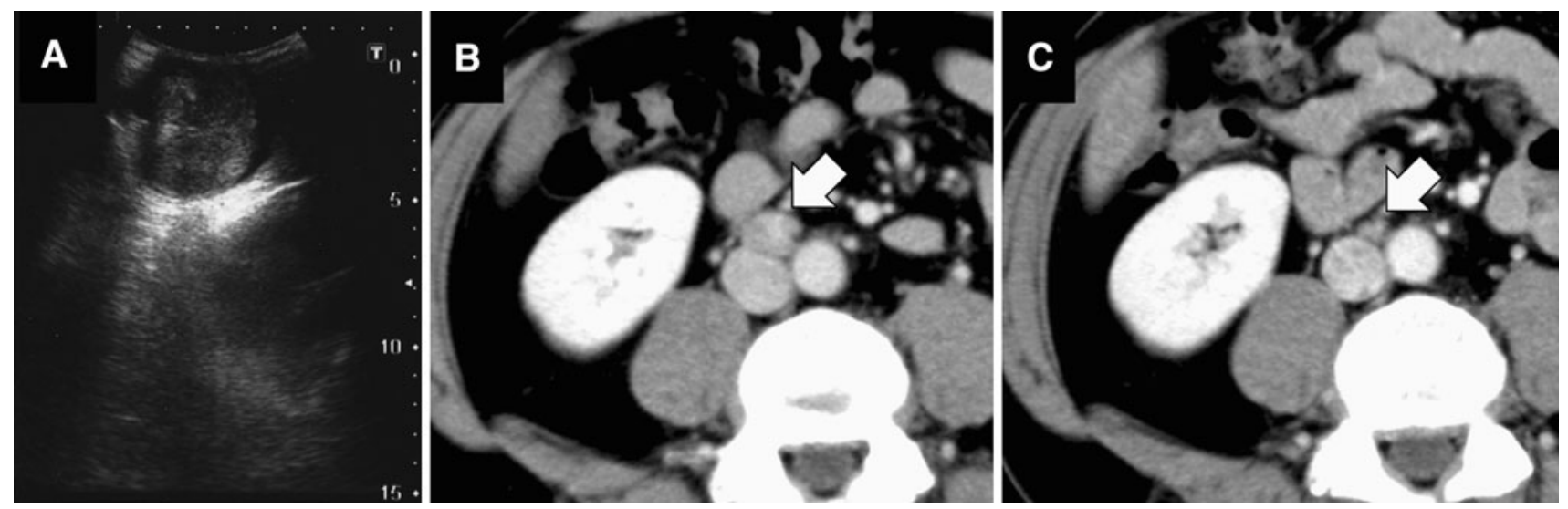

Fig. 1 Ultrasound appearance of the primary tumor and CT findings of the retroperitoneal lymph node metastases. Ultrasound examination showed a heteroechoic tumor in the right testis (a). CT scans revealed retroperitoneal lymph node metastases (b), which showed remarkable reduction after 4 cycles of VIP (c)

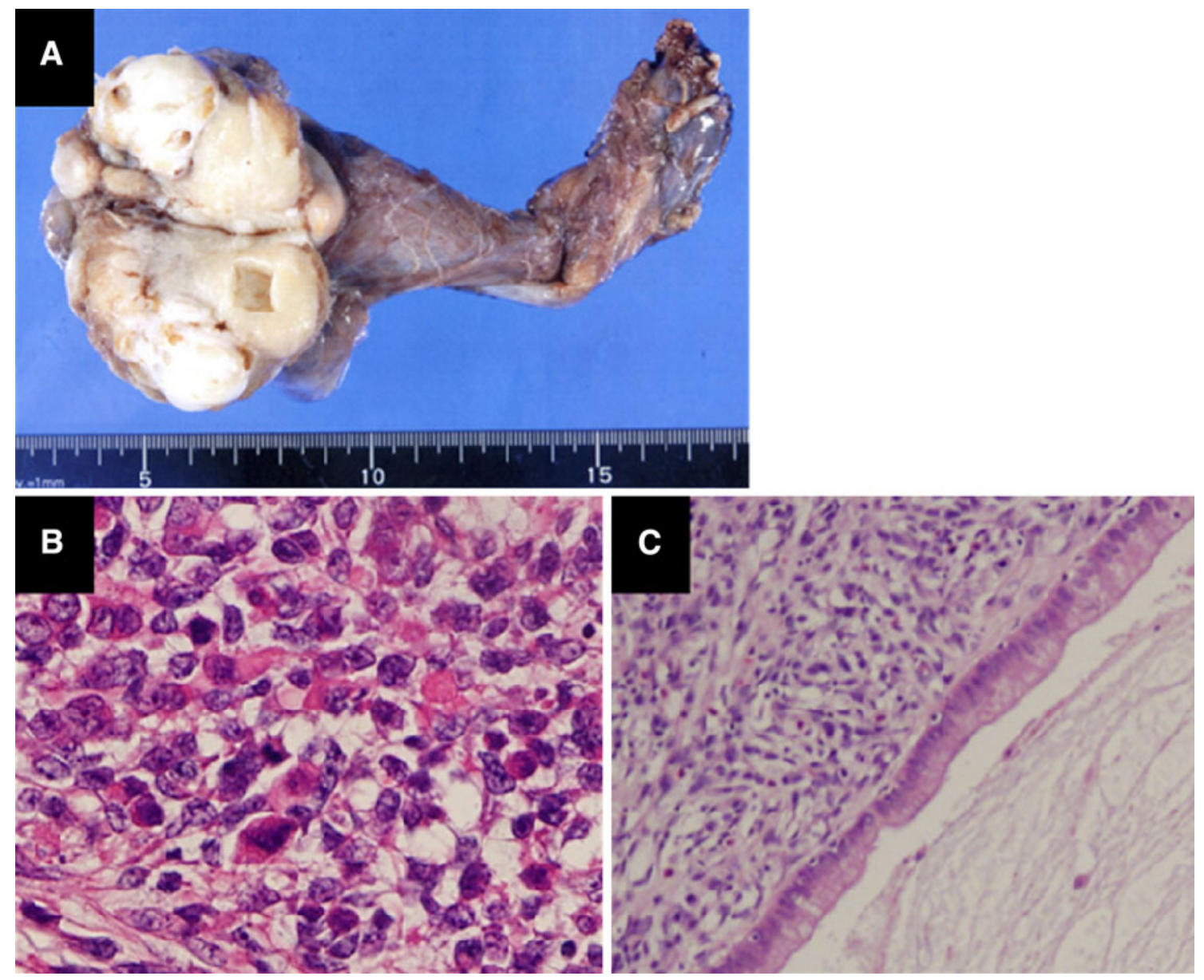

Fig. 2 Macroscopic and histological findings of the primary tumor $(H \& E, \times 400)$. Tumor showed a solid white to yellow cut surface with focal areas of hemorrhage (a). MT component consisted of small

both small round tumor cells with hyperchromatic nuclei and large polygonal-shaped tumor cells with abundant eosinophilic cytoplasm (Fig. 2b), while the remaining round cells and large polygonal-shaped cells with abundant eosinophilic cytoplasm (b). Mature teratoma element consisted of columnar epithelia with goblet cells (c)

lesion was a mature teratoma consisting of columnar epithelial cells with goblet cells (Fig. 2c). Immunohistochemical examination (Fig. 3) revealed that the non-teratoma 

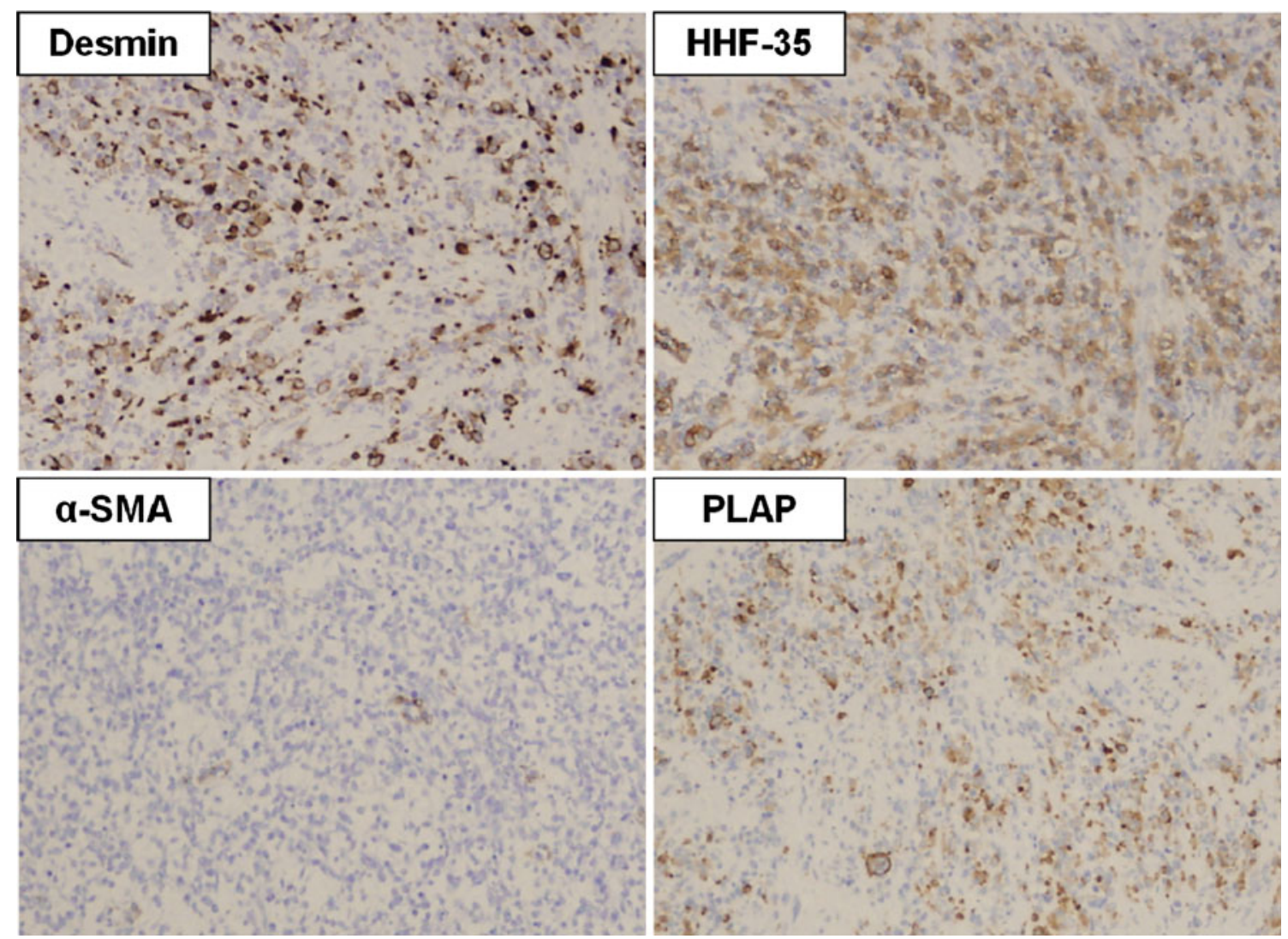

Fig. 3 Immunohistochemical findings of the MT element in the primary tumor $(H \& E, \times 100)$. MT cells were positive for desmin, HHF-35, and PLAP, but negative for $\alpha$-SMA

component of the tumor was positive for muscle-specific actin (HHF-35) and desmin and negative for $\alpha$-smooth muscle actin ( $\alpha$-SMA); accordingly, this component was diagnosed as rhabdomyosarcoma $[9,10]$. This rhabdomyosarcoma cells concomitantly expressed placental alkaline phosphatase (PLAP), which is a marker of primitive germ cells and GCTs $[11,12]$.

On the basis of these immunohistochemical findings which suggested that the rhabdomyosarcoma component arose via the MT of preexisting GCTs, we applied GCToriented chemotherapy including etoposide, ifosfamide, and cisplatin (VIP) [13] as first-line therapy. As there was a concern about tumor progression during the chemotherapy due to a chemoresistance of the MT element or growing teratoma syndrome [14], we were prepared to change the chemotherapy protocol or perform salvage surgery in case of a progression. However, the retroperitoneal mass showed continuous shrinkage with normal serum marker levels. After 4 cycles of VIP, the patient achieved a radiological partial response in the retroperitoneal lymph nodes (Fig. 1c). The patient underwent retroperitoneal lymph node dissection. The resected specimen included 9 lymph nodes, which were histologically necrosis and fibrosis without any viable teratomatous or other GCT cells. Sixteen months after the operation, the patient remains in good condition with no evidence of recurrence.

\section{Discussion}

This is a rare case of a testicular rhabdomyosarcoma with teratoma, in which complete remission of retroperitoneal metastases was achieved by GCT-oriented chemotherapy. Although the MT element has been commonly regarded to be chemoresistant [5], this case suggests the therapeutic potential of GCT-oriented chemotherapy in management of metastatic GCT with MT. Considering the immunohistochemical findings which revealed the coexpression of a GCT marker (PLAP) within the rhabdomyosarcoma element in the present case, it is suggested that GCT-oriented chemotherapy may be more useful in patients with a tumor which shows a coexpression of markers for MT and GCT.

The MT of GCT remains rare and poorly understood. Although this phenomenon is often designated as 'teratoma with MT', it can occur without teratoma in original GCT lesions: in fact, the absence of teratomatous elements has been reported in $10-45 \%$ of cases $[2,5-8,15,16]$. To date, two mechanisms for the development of MT have been 
postulated: malignant differentiation of the totipotential embryonal carcinoma cells, or malignant 'de-differentiation' of mature teratoma elements [3, 4]. Although these phenomena can be induced by chemotherapy [2] or radiotherapy [17], they can occur naturally without any clinical induction [18]. The presence of MT element in the primary chemo-naïve tumors, which is also observed in the present case, suggested that MT can occur early during tumor pathogenesis. In the recent study by Necchi et al. [19], MT occurred in the primary tumor in $17(35 \%)$ patients, and was diagnosed at stage I in 12 (25\%) of the 48 patients with GCT with MT. The reported case of naturally occurring MT in a child also suggested that MT can occur early during tumorigenesis [20].

In the present case, coexpression of PLAP in the rhabdomyosarcoma component was observed. A previous study reported that expression of PLAP in normal testicular tissues is observed only in the primitive embryonic cells [11]. In GCTs, more than $90 \%$ of intratubular malignant germ cells, seminoma, and embryonal carcinoma cells showed expression of PLAP; however, its expression is limited in yolk sac tumor $(25 \%)$, mature $(5 \%)$ and immature $(4 \%)$ teratomas [12]. From these findings, PLAP has been considered to be a marker of undifferentiated germ cells or GCTs. In the present case, positive expression of PLAP observed in the MT element may suggest that the rhabdomyosarcoma component arose via MT of totipotential embryonal carcinoma cells, and that the MT element possess tumor characteristics similar to original GCTs. Another possibility why GCT-oriented chemotherapy was effective in the present case is that RPLN metastases had other GCT components such as seminoma. Although we cannot make a definitive conclusion at this time because of the rarity of the disease, we consider that GCT-oriented chemotherapy can be a good option for patients with MT which coexpresses PLAP.

The role of chemotherapy in multimodal treatment of metastatic GCT with MT is still controversial. Because the MT component has been thought to be resistant to cisplatin [5], several authors have already advocated the use of MTadapted chemotherapy. In a study by Donadio et al. [7], 7 of 10 patients responded initially to MT-adapted chemotherapy, but only 3 of them eventually survived. El Mesbahi et al. [8] reported that 4 of 8 patients who were heavily pretreated with GCT-oriented chemotherapy achieved partial response through MT-adapted chemotherapy, although all of them eventually progressed and died. The administration of broad-spectrum agents that should be effective against the non-GCT components seems logical, but the efficacy of MT-adapted chemotherapy has not been established. In contrast, the possible efficacy of GCT-oriented chemotherapy in multimodal treatment for GCT with MT has been suggested recently. In a study by Necchi et al.
[19], 7 of 11 patients who received GCT-oriented chemotherapy as a first-line treatment were rendered disease-free with a median progression-free survival time of 8 months, and 3 of 8 patients who received GCT-oriented chemotherapy as a salvage treatment also survived.

Though patients with metastatic GCT with MT may respond to chemotherapy, a long-term cure with chemotherapy alone is rarely met. In the current case, as there was concern about progression during chemotherapy due to the proliferation of chemoresistant MT cells or teratoma cells, we performed a CT scan after every cycle to determine whether we should perform surgery immediately or continue chemotherapy. Complete surgical removal of all residual masses is the only chance for a long-term cure and should be considered the mainstay of therapy [2, 5, 6].

In summary, we report a case of metastatic teratoma with rhabdomyosarcoma successfully treated with GCToriented chemotherapy followed by surgical resection. Although treatment for GCT with MT is still controversial, GCT-oriented chemotherapy may be a good option, particularly for patients with a tumor which coexpresses GCT markers such as PLAP.

Conflict of interest The authors declare that they have no conflict of interest.

\section{References}

1. Eble JN, Sauter G, Epstein JI et al (eds) (2004) World Health Organization classification of tumours. Pathology and genetics of tumours of the urinary system and male genital organs. IARC, Lyon

2. Ulbright TM, Loehrer PJ, Roth LM et al (1984) The development of non-germ cell malignancies within germ cell tumors. A clinicopathologic study of 11 cases. Cancer 54:1824-1833

3. Ahmed T, Bosl GJ, Hajdu SI (1985) Teratoma with malignant transformation in germ cell tumors in men. Cancer 56:860-863

4. Little JS Jr, Foster RS, Ulbright TM et al (1994) Unusual neoplasms detected in testis cancer patients undergoing post-chemotherapy retroperitoneal lymphadenectomy. J Urol 152:1144-1149

5. Motzer RJ, Amsterdam A, Prieto V et al (1998) Teratoma with malignant transformation: diverse malignant histologies arising in men with germ cell tumors. J Urol 159:133-138

6. Comiter CV, Kibel AS, Richie JP et al (1998) Prognostic features of teratomas with malignant transformation: a clinicopathological study of 21 cases. J Urol 159:859-863

7. Donadio AC, Motzer RJ, Bajorin DF et al (2003) Chemotherapy for teratoma with malignant transformation. J Clin Oncol 21:4285-4291

8. El Mesbahi O, Terrier-Lacombe MJ, Rebischung C et al (2007) Chemotherapy in patients with teratoma with malignant transformation. Eur Urol 51:1306-1311 (discussion 1311-1312)

9. Coindre JM (2003) Immunohistochemistry in the diagnosis of soft tissue tumours. Histopathology 43:1-16

10. Schmidt RA, Cone R, Haas JE et al (1988) Diagnosis of rhabdomyosarcomas with HHF35, a monoclonal antibody directed against muscle actins. Am J Pathol 131:19-28 
11. Hustin J, Collette J, Franchimont P (1987) Immunohistochemical demonstration of placental alkaline phosphatase in various states of testicular development and in germ cell tumours. Int $\mathbf{J}$ Androl 10:29-35

12. Burke AP, Mostofi FK (1988) Placental alkaline phosphatase immunohistochemistry of intratubular malignant germ cells and associated testicular germ cell tumors. Hum Pathol 19:663-670

13. Hinton S, Catalano PJ, Einhorn LH et al (2003) Cisplatin, etoposide and either bleomycin or ifosfamide in the treatment of disseminated germ cell tumors: final analysis of an intergroup trial. Cancer 97:1869-1875

14. Logothetis CJ, Samuels ML, Trindade A et al (1982) The growing teratoma syndrome. Cancer 50:1629-1635

15. Ganjoo KN, Foster RS, Michael H et al (2001) Germ cell tumor associated primitive neuroectodermal tumors. J Urol 165:1514-1516

16. Guo CC, Punar M, Contreras AL et al (2009) Testicular germ cell tumors with sarcomatous components: an analysis of 33 cases. Am J Surg Pathol 33:1173-1178
17. Radford DM, Johnson FE, Janney CG (1991) Sarcomatous change in a teratoma after treatment of testicular carcinoma. Cancer 68:395-399

18. Morinaga S, Nomori H, Kobayashi R et al (1994) Well-differentiated adenocarcinoma arising from mature cystic teratoma of the mediastinum (teratoma with malignant transformation). Report of a surgical case. Am J Clin Pathol 101:531-534

19. Necchi A, Colecchia M, Nicolai N et al (2011) Towards the definition of the best management and prognostic factors of teratoma with malignant transformation: a single-institution case series and new proposal. BJU Int 107:1088-1094

20. Takahashi M, Okumura N, Matsuoka T et al (2011) Teratoma with naturally occurring malignant transformation in a child. Ann Thorac Cardiovasc Surg (Epub ahead of print) 